\title{
Model-Driven Web Engineering Performance Prediction with Layered Queue Networks
}

\author{
Alessio Gambi ${ }^{1}$, Giovanni Toffetti ${ }^{1}$, and Sara Comai ${ }^{2}$ \\ 1 University of Lugano, \\ 6904, Lugano, Switzerland \\ \{alessio.gambi, toffettg\}@usi.ch \\ 2 Politecnico di Milano, \\ 20133, Milan, Italy \\ comai@elet.polimi.it
}

\begin{abstract}
This position paper describes an approach to predict the performances of a Web application already in the early stages of application development. It leverages the wealth of information of MDWE solutions to automatically obtain accurate representations of the running application in terms of layered queue networks (LQNs), i.e., analytical models simulating the behavior of the system and computing the performances mathematically. In particular, the paper discusses how a MDWE methodology can be exploited to generate such performance models and presents a proof of concept example.
\end{abstract}

Keywords: layered queue networks, performance, web engineering, model transformation, capacity planning, WebML.

\section{Introduction}

Being able to correctly size the resources needed by a Web application to its incoming workload is the ultimate goal of performance engineering 46]. For a company, both over- and under-provisioning turn into revenue loss: in the former case because of higher than needed operational costs, in the latter case because the Quality of Service is below clients' expectations. The current practice in performance engineering impacts on different phases of a Web application development cycle: early in the process, coarse high level specifications are used at design-time to build mathematical models (e.g., queue networks) to estimate system performances; as the application reaches a complete implementation, staged experiments provide numeric parameters for the performance models or direct measures of the complete system behaviour. Updating the mathematical models to reflect the design choices and implementation details that happen along the development cycle is a costly manual process and very seldom done, as a result performance models tend to be misaligned with the final system and their predictions are less accurate.

F. Daniel and F.M. Facca (Eds.): ICWE 2010 Workshops, LNCS 6385, pp. 25-36 2010.

(C) Springer-Verlag Berlin Heidelberg 2010 
In this paper we propose an approach to leverage the wealth of information of MDWE solutions to automatically obtain accurate representations of the running applications in terms of layered queue networks (LQNs). The advantages of this approach with respect to the common practice are manifold: 1) formal models allow for the generation of LQNs at different levels of granularity and detail, from very coarse grained all the way to the actual code produced by model transformation, 2) they do not require manual intervention to be kept in synch with the development process, 3 ) any model update can be reflected directly in LQNs providing immediate feedback on expected performance degradation/improvement. These advantages are particularly significant in the context of Web engineering, since most Web applications are constantly under maintenance and evolution.

The paper is structured as follows: Sections 2 and Section 3 provide an overview of performance engineering and LQNs; Section 4 discusses how a MDWE methodology can be exploited to generate performance models. Section 5 presents a proof of concept example. Finally, Section 6 presents related work and Section 7 draws the conclusions.

\section{Performance Engineering}

Performance engineering deals with all the tools, practices, roles, and activities, that are applied to a system throughout its life-cycle in order to meet a set of non-functional (performance) requirements.

The first step of performance engineering consists in the identification of a) a set of performance objectives for a system given a well-specified set of running conditions (e.g., response time for operation X will be within 2 seconds $95 \%$ of the time under a median load of 100 concurrent clients) and b) the methods and plans to test them at staging time and monitor them in production. Then, design and development are conducted following performance and monitoring requirements (e.g., by considering task parallelism, by adding instrumentation to the code for monitoring, etc.). At the end of the design phase, an high-level representation of the main components can already be used to do preliminary performance analysis.

Analysts normally utilize a combination of analytical model, simulation model, and empirical analysis based techniques [1. Analytical models can be used in the early phases of development, and they represent the system behaviour by means of mathematical equations. The solution of these equations results in the values of the performance metrics. Analytical models are generally fast to solve, however the set of the underlying simplifying assumptions and the tuning of their parameters greatly impact on their accuracy. Simulation models provide more accurate results but at higher costs. Those models represent the system behavior as a chronological sequence of events, where each event occurs at an instant in time and changes the state of the system. During the simulation statistical techniques are used to compute the values of performance metrics. Finally, empirical analysis returns the most accurate performance measures, since the actual system implementation is stressed with different workloads. 
In this work, we concentrate on analytical models (in particular LQNs) and propose a wider adoption of these performance modeling solutions in the context of MDWE. The rationale behind our proposal is that, given a knowledge or representation of the code generation mechanism, the design phase in MDWE already provides a complete representation of the final system that can be combined with a deployment description to produce accurate performance models.

\section{Layered Queue Networks}

Queue network (QN) models are one of the main tools supporting performance engineering and are used to determine (as average values) important metrics such as response time, system throughput, and resource utilization [1. QNs represent systems as collection of resources, called service centers, that multiple clients need to access. Clients compete to access resources and their contention is represented by means of queueing before the service center. The time spent by a service center to complete a client request is called service time $(\mathbf{s})$, and the idle time spent by a client between two different requests is called think time $(\mathbf{z})$. In real systems, a client request needs to access multiple resources, so requests in the model go through different service centers and queues. For example, an HTTP request for a dynamic page starts from the Browser, and it may lead to several requests to the Front End and the Back End servers. This example is depicted in the form of a sequence diagram in Figure 1 ta. Notice that, during the computation, the browser is blocked waiting for the front end while the front end is waiting for the back end; this situation impacts on the final service time and it must be taken into account while computing the performance metrics.

This situation can be accurately modeled by means of a particular kind of QN model, called the layered queue network (LQN) [11]. LQN models extend plain queue networks and combine architectural information with consumption and load data; in this way, LQNs can capture the nesting of resources in the

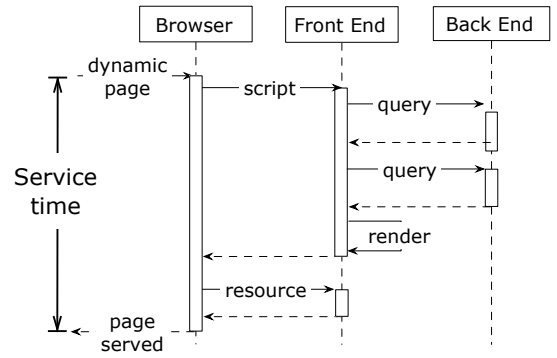

(a)

$\longrightarrow \begin{aligned} & \text { synchronous request } \\ & \text { reply }\end{aligned}$

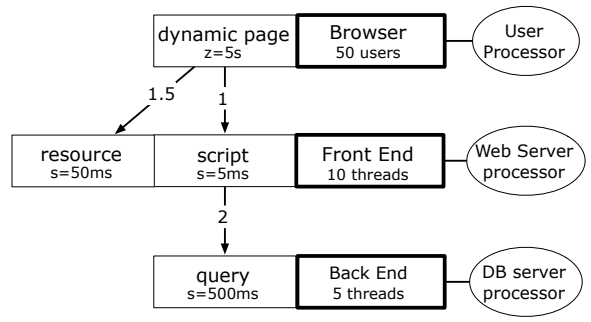

(b)

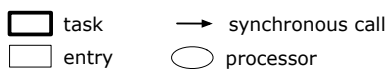

Fig. 1. A sequence diagram modeling a dynamic page request (on the left) and an LQN that models it (on the right) 
system and their effects on the performance metrics. In the LQN notation, service centers are represented by tasks that may offer different services (or operations), which are modeled by means of entries. Each task has its own queue that is shared among the all requests for its different entries, and it is associated to a processor, which models the "physical" entity running it. Tasks may specify a multiplicity value to model their multi-server nature, and entries specify the service demand, which is the cost of running them on the associated processor. If an entry models a user activity then the think time is specified instead of the service demand. Entries can request services to entries of other tasks (represented by calls); calls may be synchronous (and therefore blocking) and can specify the mean count of requests per operation.

The LQN in Figure 1 1 b models a multi tiered system used to provide dynamic pages. The LQN contains three tasks, Browser, Front End and Back End, that are deployed on three different processors, respectively User, Web server and DB server processors. There are 50 concurrent users that request generic dynamic pages to the browser, with an average think time of 5 seconds. At the Front End task, each user request generates an average of 1.5 blocking requests for static resources and 1 blocking request for the dynamic content (the script entry). Static resources are entirely served by the Front End, while to complete the script entry, 2 blocking requests are additionally issued at the Back End (query entry). The LQN specifies also multiplicities and service demands.

Layered queue networks are a powerful tool that can support the different phases of performance engineering. They can describe systems at different levels of abstraction: for example, tasks can be used to model software entities and to find software bottlenecks; entries can be used to model aggregate requests, at page level, or they can be further refined using activities; activities allow to model complex flows with loops, forks, and other constructs. For example they can be used to model accurately all the steps that an application server performs while building and rendering a dynamically generated page.

\section{Model-Driven Performance Prediction}

In this paper we advocate the usage of MDD for the generation of performance models at different levels of accuracy. For the sake of the exposition, we will use the Web Modeling Language (WebML) [2] as a reference language, since it is both well known in the community, and its runtime architecture is fairly straightforward. We stress however, how the process is general and can therefore be applied to any MDWE methodology.

As introduced in the previous section, a LQN uses primitives representing both the user behavior and the high-level system internal functioning. In particular, considering their main drivers, we can separate a LQN in different parts:

1. The tasks, entries, activities and request types that depend on the design of the application and architecture;

2. The hosts and tasks multiplicity that depend on the final deployment;

3. The think time and average request mix that depend on the user behavior; 
4. The resource usage and configuration parameters that can either be obtained by system experts or measured with specific experiments on the system implementation.

In the following subsections, we discuss how, given a Web Engineering model with formal semantics and a known architecture (like, e.g., WebML/WebRatio), various queue models focusing on different aspects can be automatically obtained; an example of LQN derived from WebML/WebRatio will be provided in Section 5 .

\subsection{LQN Modelling Choices for the Model/Architecture}

Figure 2 gives a high-level representation of the runtime architecture of a WebML application in WebRatio [2]. On the left-hand side of the figure, users make HTTP requests through their browser. HTTP requests are served by a Web server (e.g., Apache): static resources are directly served, while dynamic pages are forwarded to a load-balanced pool of application servers or servlet containers. Each servlet container runs an implementation of Struts, a common MVC2 framework for the Web. The framework controller forwards the requests to a specific action (page or operation), which in turn invokes the WebRatio runtime components (RTX) to compute the page along with its content units by querying one or more databases.

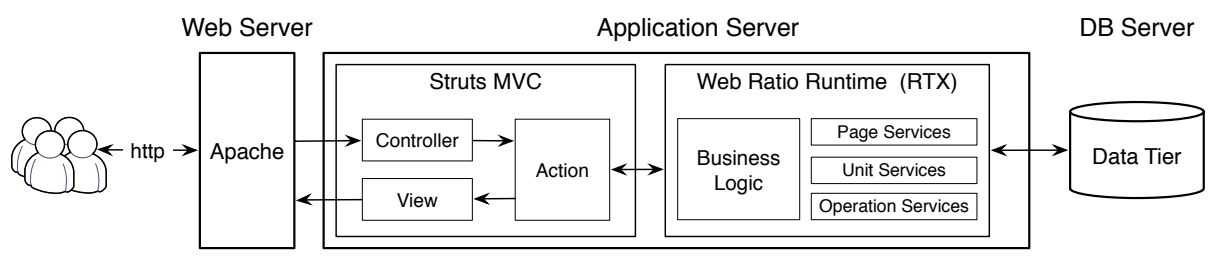

Fig. 2. High-level representation of the architecture of a WebML/WebRatio application

The previous architecture would be enough to create a simplified queue model reflecting the system layers, but delving deeper into the architecture's internals can provide more useful information. To serve dynamic WebML pages the RTX Page Service performs a different computation sequence depending on the link navigated by the user (for example, Link $\mathrm{X}$ in Figure 3 ). The page computation populates all the content units along with a link-specific parameters propagation to provide the correct query parameters to the database. Hence, each page computation is different and requires a computation time that is at least proportional to the number of units it contains. Our practical experience shows that the number of content units alone is a good metric of the response time of a dynamic page as this relates to the load at the database plus the waiting time for querying it. 


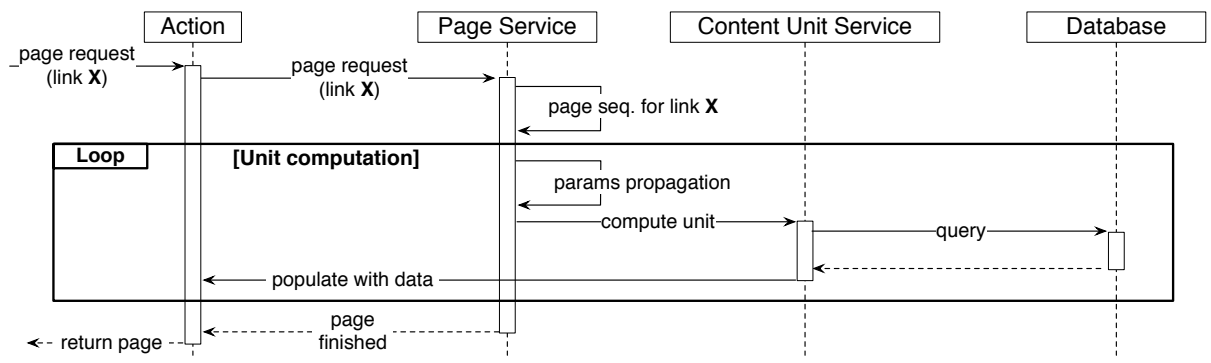

Fig. 3. Simplified sequence diagram of page computation

Since each link may trigger a different page computation sequence, even at the level of a single page the system may face a different load depending on the navigated link. For example, in WebML some units are not populated until they receive parameters through a normal (i.e. non-automatic) link, thus their computation does not always involve database operations. The model-to-model transformation to translate WebML to LQNs models can be summarized as follows: to model the general WebML/WebRatio architecture in LQN terms, we define the App Server task, representing the servlet container, and the DB server task, i.e., the data tier logic. Both tasks are application independent: they would be present in the LQN model of any WebML application. To model the application-specific logic, inside the App Server task we define an entry for each navigation link, then we create an activity for each content unit and operation, and finally we link all the activities according to page computation sequences. Each activity in the App Server task is connected to a corresponding DB server entry by means of synchronous calls.

At the DB server task, entries are mapped to SQL statements according to their functionality: for example, units with simple (attribute) selectors are mapped to plain selects, while units with selectors based on relationships are mapped to queries with joins.

Figure 4 depicts a simple WebML model fragment (on the left side) and the LQN sub-model obtained by applying the model transformation (on the right).

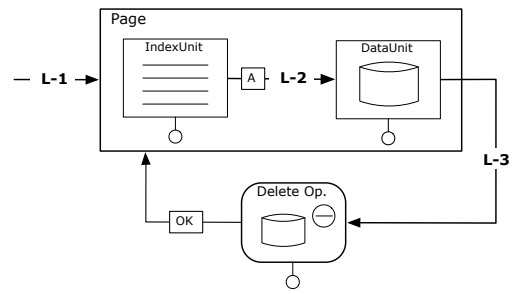

(a)

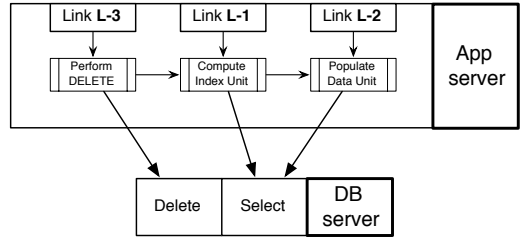

(b)

Fig. 4. A simple WebML hyperlink model (a) and the corresponding LQN model (b) 
Considering this example, if a user navigates to the page using the noncontextual link L-1, the RTX framework has to compute the index unit and then (L-2 is an automatic link) the data unit, resulting in two select operations on the DB. The LQN model reflects this behaviour using the entry named Link L-1 and the sequence of activities: Compute Index Unit and then Compute Data Unit. If the user navigates to the page using link L-2 instead, the computation sequence changes and only one select on the DB is required. Again the LQN reflects this situation using a different entry (Link L-2) connected only to the last activity. Finally, if the user issues the delete operation (link Link L-3) the runtime framework has to perform the delete operation first, and then it must recompute the whole page (as in the first case). The LQN models this using an additional entry triggering the sequence of all three activities. If we were to know with sufficient detail the typical user navigation pattern, we could predict with good accuracy the system load and the average response times for each page for a varying number of concurrent clients. Moreover, taking full advantage of the wealth of knowledge an application model contains, one could use additional information to estimate the computation time of each unit in a page just considering its type, multiplicity, and selectors and how they impact on query execution, state object population, and rendering time (e.g., selectors on relationship imply joins, hierarchical index units have a query with join per each level).

Clearly, the more complex the LQN, the more parameters are needed and the longer it takes to be computed. A possible measure to reduce LQN complexities is to sacrifice some detail by aggregating all the activities in a single entry per page, or by aggregating all units per type, providing a reference execution time per each type at the DB Server task.

\subsection{User Behavior and Resource Usage}

Two important parts of the LQN cannot be directly derived from the application models as they are: the user navigation model and an estimate of the resource usages (e.g., the time spent for computing a specific unit on a specific platform).

Concerning the former case the model can provide us, through transformations, with a representation of all possible navigation steps (e.g., a Markov chain with probability equally distributed among all navigable links in a page). But real applications have specific user roles with their own common navigational behaviour. These behaviours have to be extracted from real application scenarios (usually collected at beta application release) and represented consistently in the LQN using a combination of activities, OR-forks, and probability. An example of such user LQN is shown in the top part of Figure 6 where a user can choose among three different navigation paths.

With respect to resource usage, the LQN model parameters have to be set according to the experience of a system expert or have to be measured with ad-hoc experiments at staging time. In a possible future scenario, WebRatio could provide designers with a set of benchmark resource needs for units and deployments gathered from real applications. 
Given user navigation paths and resource usages, the LQN will provide insightful information to an application designer in two different ways. First of all, it can be computed in the background (possibly at each model modification) at design time or during site evolution to provide immediate feedback on the expected response time of all application pages. This will indicate to the developer possible alternative designs, for example by suggesting to break a complex page into multiple navigational steps. On the other hand, at deployment time, the LQN can be used to provide prediction on the optimal tuning of different architectural configuration parameters (e.g., number of DB connections, number of threads in the AS) by manually setting them in the model and performing an exploration in the configuration parameter space.

\section{A Proof of Concept Example}

As a proof of concept, we will use a product catalogue and content management system of a small furniture manufacturing company, called Acme, to generate LQN models and discuss results. An excerpt of hypertext model is shown in Figure 5. We used a simple XSL stylesheet to transform the application model for the public siteview of Acme into a LQN representation for lqns (Layered Queueing Network Solver and Simulator 1 ).

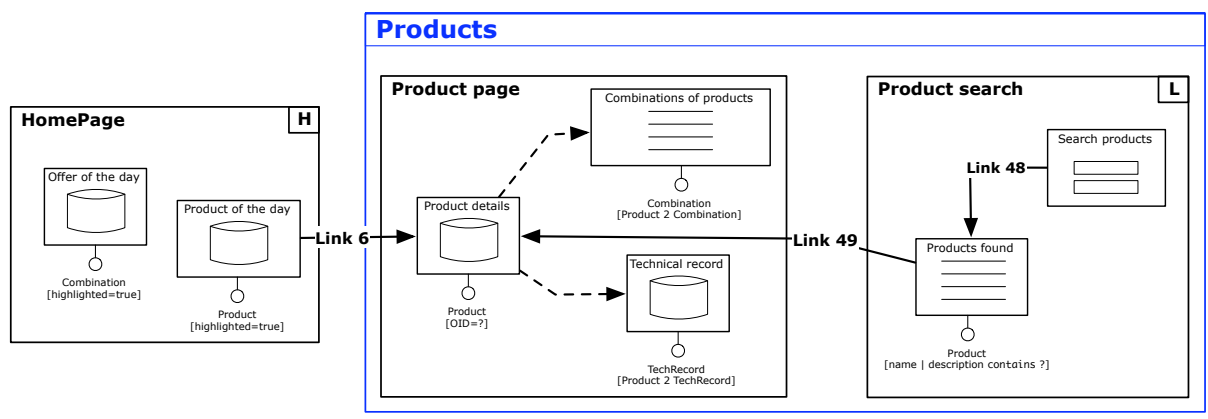

Fig. 5. Excerpt of the hypertext model for the Acme application

Each page computation sequence has been transformed into a sequence of activities at the application server level; we defined a simple non-intense 2 navigation sequence for users (directly coded in the XSL), assigned a common resource usage for page and unit rendering (10ms each), and different db resource usages for queries (10ms for selects, $20 \mathrm{~ms}$ for selects with joins) 3 . Concerning the architecture, we are using a simplified solution with one application server (AS)

\footnotetext{
1 http://www.sce.carleton.ca/rads/lqns/

2 Users have 3 possible navigational paths asking around 5 pages over 20 seconds and repeat the cycle every 2 minutes.

3 The resource usages we chose are realistic but have no significance, our aim is to provide a numerical example.
} 


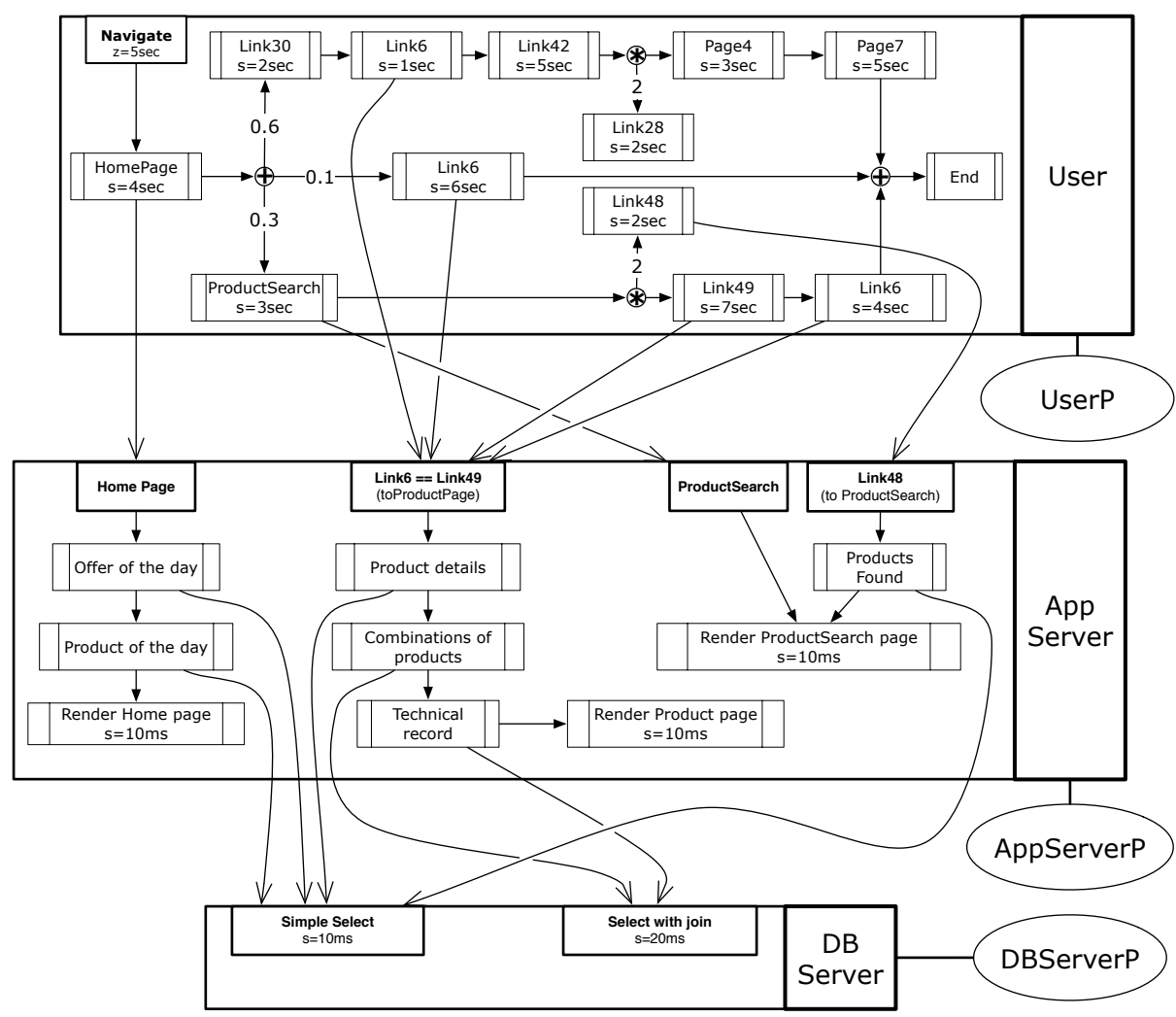

Fig. 6. A partial representation of the LQN for the Acme case study (limited to the Hypertext model in Figure 5]

and one database (DB) running on separate machines. A partial representation of the LQN we used is shown in Figure 6.

Figure 7 shows the plot of the predicted response times for pages Home (page1), Product Details (page2), Product Search (page19) and performing a search (ln48) in the Acme public siteview under varying user load with a fixed system configuration of 20 threads both on the AS and the DB. In the same graph, we also plotted the predicted CPU utilizations for both AS and DB. The reader should notice how as soon as the AS CPU is close to saturation response times raise abruptly. The bottleneck for this load and configuration is therefore the AS, while the DB could sustain higher load.

In Figure 8 instead, we use the LQN to explore one of the AS configuration parameters, in particular we try to identify the optimal number of threads to use under different numbers of concurrent clients. Our interpretation of the results is that for high loads having more that 10 threads has negative effects, while for low loads the differences in response times are minimal. 


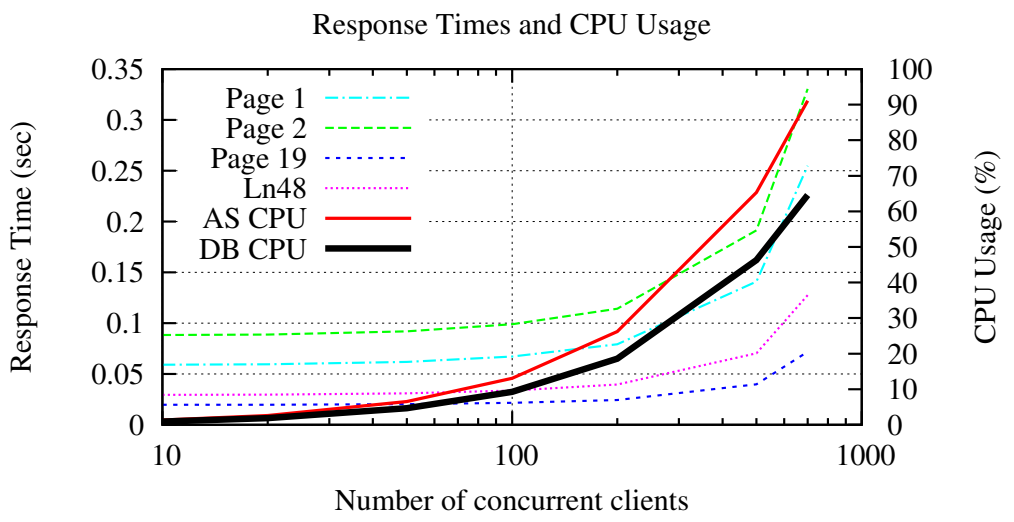

Fig. 7. LQN prediction of response times for two pages and CPU utilization for a synthetic workload

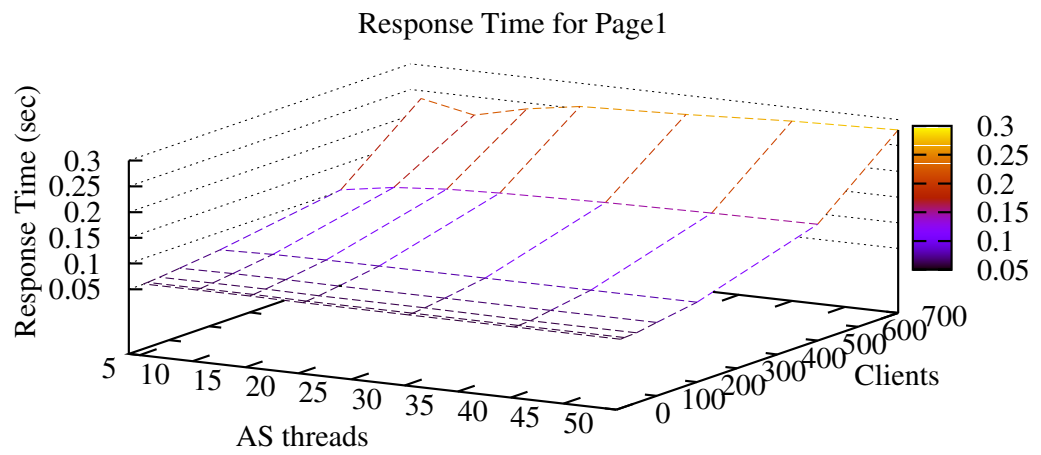

Fig. 8. LQN prediction of response times for Page1 varying concurrent clients and AS threads

\section{Related Work}

The concept of Model-Driven Performance Engineering was introduced by Frietzsche and Johannes in [6] and is defined as a process combining MDE and performance engineering: structural and behavioral models can be annotated with performance information and performance analysis can be applied at the different stages of refinement of the MDE process. In this context, several works have been proposed, typically considering UML design models that are transformed into a LQN or queueing network performance model, like, for example, [74] where XLST and ATL rules are used for the transformation, respectively. Compared to these works our approach is quite similar, since we translate pieces of WebML into LQN sub models and we use LQN for the exploration of parameters. 
However, starting from a Web modeling language, we can choose different levels of details/abstraction specifically tailored to Web applications.

LQNs are one of the most adopted technique and have been efficiently applied also in other related contexts, like ERP applications [10] heavily adopting multithreading and multi-core architectures, in the early phases of Software Product Lines [12, in the optimization of the deployment of multiple Web applications on a cloud [8], with replicated components [9], where LQN based approaches scale very well.

Considering the Web Engineering context, the problem of performances has been treated in different works: [3] discusses techniques for balancing load among multiple servers and shows how Web pages can be designed to improve performances; [5] shows a technique to minimize the access cost of a website by adding hotlinks that make the most accessed pages more accessible. In all these cases no formal models are employed and no performance prediction is applied.

In [13] a model-driven capacity planning tool suite, called Revel8or, for J2EE, .Net and Web services platforms is proposed to analyze and predict Web/Web services performance: this tool integrates performance analysis based on UML and Queueing Networks, with automated benchmark generation. Compared to this suite, our proposal goes in to deeper detail in the analysis of models specifically designed for Web applications and, to the best of our knowledge, this is the first time that LQN are obtained from a Web Engineering modeling notation.

\section{Conclusions}

In this paper we have described a model-driven approach that derives LQN models for the performance prediction of a Web application from a MDWE methodology. A proof of concept applied on WebML/WebRatio is presented. Although much work must be done to validate the applicability of the whole approach in practice, on the basis of this experience we retain that MDWE techniques can be efficaciously employed to automatically obtain accurate representations of the running applications behaviour. As future work we plan to apply the proposed approach to real case studies and to implement a framework for WebML/WebRatio based on LQNs.

Acknowledgments. We wish to thank Antonio Carzaniga for his comments and suggestions. This work is partially supported by the European Community under the IST programme of the 7th FP for RTD - project RESERVOIR contract IST-215605.

\section{References}

1. Balsamo, S., Di Marco, A., Inverardi, P., Simeoni, M.: Model-based performance prediction in software development: A survey. IEEE Trans. Softw. Eng. 30(5), 295310 (2004)

2. Ceri, S., Fraternali, P., Bongio, A., Brambilla, M., Comai, S., Matera, M.: Designing data-intensive Web applications (2003) 
3. Challenger, J., Iyengar, A., Dantzig, P., Dias, D.M., Mills, N.: Engineering highly accessed web sites for performance. In: Murugesan, S., Desphande, Y. (eds.) Web Engineering. LNCS, vol. 2016, pp. 247-265. Springer, Heidelberg (2001)

4. Cortellessa, V., Di Gregorio, S., Di Marco, A.: Using atl for transformations in software performance engineering: a step ahead of java-based transformations? In: WOSP 2008: Proceedings of the 7th international workshop on Software and performance, pp. 127-132 (2008)

5. Czyzowicz, J., Kranakis, E., Krizanc, D., Pelc, A., Martin, M.V.: Enhancing hyperlink structure for improving web performance. J. Web. Eng. 1(2), 93-127 (2003)

6. Fritzsche, M., Johannes, J.: Putting performance engineering into model-driven engineering: Model-driven performance engineering. In: Engels, G., Opdyke, B., Schmidt, D.C., Weil, F. (eds.) MODELS 2007. LNCS, vol. 4735, pp. 164-175. Springer, Heidelberg (2007)

7. Gu, G.P., Petriu, D.C.: Xslt transformation from uml models to lqn performance models. In: WOSP 2002: Proceedings of the 3rd international workshop on Software and performance, pp. 227-234 (2002)

8. Li, J.Z., Chinneck, J., Woodside, M., Litoiu, M., Iszlai, G.: Performance model driven QoS guarantees and optimization in clouds. In: Proceedings of the ICSE Workshop on Software Engineering Challenges of Cloud Computing, pp. 15-22 (2009)

9. Omari, T., Franks, G., Woodside, M., Pan, A.: Solving layered queueing networks of large client-server systems with symmetric replication. In: Proceedings of the International Workshop on Software and performance (WOSP 2005), pp. 159-166 (2005)

10. Rolia, J.A., Casale, G., Krishnamurthy, D., Dawson, S., Kraft, S.: Predictive modelling of sap erp applications: challenges and solutions. In: Proceedings of the International ICST Conference on Performance Evaluation Methodologies and Tools, pp. 1-9 (2009)

11. Rolia, J.A., Sevcik, K.C.: The method of layers. IEEE Transactions on Software Engineering 21, 689-700 (1995)

12. Tawhid, R., Petriu, D.C.: Integrating performance analysis in the model driven development of software product lines. In: Czarnecki, K., Ober, I., Bruel, J.-M., Uhl, A., Völter, M. (eds.) MODELS 2008. LNCS, vol. 5301, pp. 490-504. Springer, Heidelberg (2008)

13. Zhu, L., Liu, Y., Bui, N.B., Gorton, I.: Revel8or: Model driven capacity planning tool suite. In: ICSE 2007: Proceedings of the 29th international conference on Software Engineering, pp. 797-800 (2007) 